\section{Graduierung von Sideropneumokoniosen}

Zusammenfassung: Durch pathologisch-anatomische Untersuchungen von Biopsie- und Autopsiepräparaten von 43 Männern mit anamnestisch gesicherter beruflicher Schweißrauchexposition waren bei 38 Proben $(88 \%)$ charakteristische Veränderungen verschiedener Schweregrade von Sideropneumokoniosen zu dokumentieren. Anhand wiederkehrender histologischer Befunde der Vermehrung und Aktivierung von Eisen-speichernden Makrophagen (Siderophagen) und variabel ausgebildeter Fibrosierungen wird eine Einteilung der Sideropneumokoniosen in drei Schweregrade vorgenommen. Dabei sind durchaus intraindividuell und auch toporegional unterschiedliche Schweregrade vorhanden. Nach morphologischen Kriterien der drei Stadien konnten sieben Präparate dem Stadium I, 21 Befunde dem Stadium II und 10 Präparate dem Stadium III zugeordnet werden. Die Ergebnisse zeigen, dass Schweißrauchexpositionen in Abhängigkeit von Dauer, Ausmaß und besonderen Umständen nach pathologisch-anatomischen Befunden durchaus zu Lungenveränderungen von Krankheitswert führen. Eine für gutachterliche Fragestellungen verbindliche Aussage über den Grad der Funktionsstörungen kann beim Bild der sogenannten Schweißerlunge allerdings nur in Kombination von histologischen Befunden, detaillierten Angaben zur beruflichen Vorgeschichte und klinischen Funktionsparametern getroffen werden.

Gradation of Sideropneumoconioses: Pathological-anatomical analyses of biopsy and autopsy samples of 43 men with anamnestically established occupational exposure to welding fumes showed in 38 specimens $(88 \%)$ characteristic alterations of varying degrees of sidero pneumokonioses. Based on recurrent histological findings of increase and activation of iron storing macrophages (siderophages) and varying degrees of fibrosis, sidero pneumokonioses are classified into three grades. Intraindividually and also toporegionally different degrees may well be present. Based on morphological criteria of the three grades, seven samples were classified as grade I, 21 specimens as grade II, and 10 samples as grade III. The results show that exposure to welding fumes may well cause pulmonary alterations reaching an impairment level, depending on length, extent and special circumstances of the respective exposure. In cases of so-called welders lungs, an obliging statement in an expert's opinion on the degree of functional impairment, however, can only be given when histological findings, detailed information on the occupational history and clinical functional parameters are combined.

Pneumologie 2000; 54: 315-317

(C) Georg Thieme Verlag Stuttgart · New York ISSN 0934-8387
K. M. Müller, M. A. Verhoff

Institut für Pathologie an den Berufsgenossenschaftlichen Kliniken Bergmannsheil - Universitätsklinik der Ruhr-Universität Bochum

\section{Einleitung}

Morphologische Befunde über Reaktionsmuster nach unterschiedlich intensiven und langen, in der Regel beruflich bedingten Schweißrauchexpositionen der Lungen sind wiederholt beschrieben worden [1-3]. Objektivierte Funktionseinschränkungen der Lungen von wesentlichem Krankheitswert und ein daraus abzuleitender begründeter Verdacht von Berufskrankheiten sind allerdings nur selten dokumentiert [4-6 u.a.].

Führende histologische Befunde sind Anreicherungen von siderophilen Pigmenten und Eisenoxid in den Lungen, vorwiegend gespeichert in Siderophagen. Die Speicherstrukturen stellen sich in der HE- und EvG-Färbung braun und schwarz, in der Berliner-Blau-Färbung grobkörnig tief blau dar. Zusätzlich sind körnige schwarze Eisen-3-Oxidspeicherungen charakteristisch $[2,7]$.

Als weitere morphologisch fassbare Veränderungen sind variabel entwickelte Fibrosierungen bevorzugt im perivasalen und peribronchialen, in vorgeschrittenen Stadien auch alveolarseptalen Bindegewebe vorhanden. Nach Überschreiten der Selbstreinigungsmechanismen der Lunge werden die Partikel des Schweißrauches von Alveolarmakrophagen phagozytiert. Die anorganischen Partikel führen zu einer Zytolyse der Alveolarmakrophagen mit „Recycling“ der freigesetzten Fremdstoffe durch Zellen des Monozyten-Makrophagen-Systems. Im Rahmen dieses Prozesses werden Mediatorstoffe wie Interleukin 1, Tumornekrosefaktor, Fibronektin etc. freigesetzt. Die Aktivierung und Proliferation von Fibroblasten führen zur Matrix- und Kollagenfasersynthese mit Entwicklung variabler Fibrosierungen im Bereich der inkorporierten Fremdsubstanzen $(2,3,8,9]$.

Basierend auf den mikroskopisch fassbaren pathologischen Befunden wurde die Frage geprüft, ob basierend auf qualitativ und quantitativ fassbaren Veränderungen der Lungen unterschiedliche Schweregrade (Stadien) von Sideropneumokoniosen nach unterschiedlichen Schweißrauchexpositionen zu dokumentieren sind.

\section{Material und Methoden}

Untersucht wurden Gewebsproben von Biopsie- und Autopsiegut von 43 Patienten mit anamnestisch gesicherten, allerdings individuell unterschiedlichen beruflichen Schweißrauchexpositionen. Ausschlusskriterien waren unter Berücksichtigung der verfügbaren Unterlagen Hinweise auf chroni- 
sche Herzerkrankungen sowie andere wesentliche Grunderkrankungen der Lungen mit rezidivierenden Blutungen und Tumorerkrankungen. Für die mikroskopische Aufbereitung stand von 26 Patienten transbronchial und von 17 Patienten thorakoskopisch gewonnenes Untersuchungsgut zur Verfügung. In drei Fällen lagen zusätzlich die im Rahmen einer Obduktion entnommenen Lungenpräparate vor. An den Schnittpräparaten wurden HE-, Elastica van Gieson- und Berliner-Blau-Färbungen durchgeführt.

\section{Ergebnisse}

In 38 Präparaten der 43 Patienten nach anamnestisch bekannter Schweißrauchexposition sind intraalveoläre und zum Teil auch interstitielle Anreicherungen von Siderophagen mit toporegional unterschiedlich deutlicher Ausprägung zu dokumentieren. Innerhalb desselben Schnittes gibt es Areale mit nur geringgradiger Siderophagenanreicherung und Bereiche mit erheblicher Makrophagenaktivierung. Bevorzugt in den Gebieten mit starker Siderophagenanreicherung sind variabel Fibrosierungen bevorzugt in Bereichen des perivasalen, peribronchialen aber auch alveolarseptalen Bindegewebes der Lungen vorhanden.

Trotz der starken Variabilität der Befunde wird unter Berücksichtigung der qualitativ und quantitativ erhobenen Befunde in Anlehnung an die Stadieneinteilung der Pneumokoniosen
[8] eine Graduierung von drei Schweregraden der Schweißerlunge folgendermaßen vorgeschlagen (Abb.1 a, b bis $\mathbf{3 a , b}$ ):

\section{Grad I:}

Vorwiegend alveoläre aber auch interstitielle, herdförmig betonte Ansammlungen von Makrophagen, die neben Siderin feinkörniges Eisen-III-oxid und in geringem Umfang Mischstaubpartikel speichern (sog. Siderophagen). Nur wenige Makrophagen und Mischstäube im peribronchialen, perivasalen und pleuralen Bindegewebe.

Ausschließlich mikroskopisch fassbare diskrete Fibrosierungsreaktionen (Abb.1 a,b).

\section{Grad II:}

Verstärkte Anreicherungen von aktivierten Makrophagen und Mischstaubpartikeln in perivasalem, broncho-pulmonalem und paralymphatischem Bindegewebe und in der Pleurahauptschicht.

Deutliche Fibrosierungen im Bereich der Staubdepots.

Diskrete unspezifische entzündliche Begleitreaktion (Abb. 2a,b).

\section{Grad III:}

Ausgeprägte Mischstaubdepots.

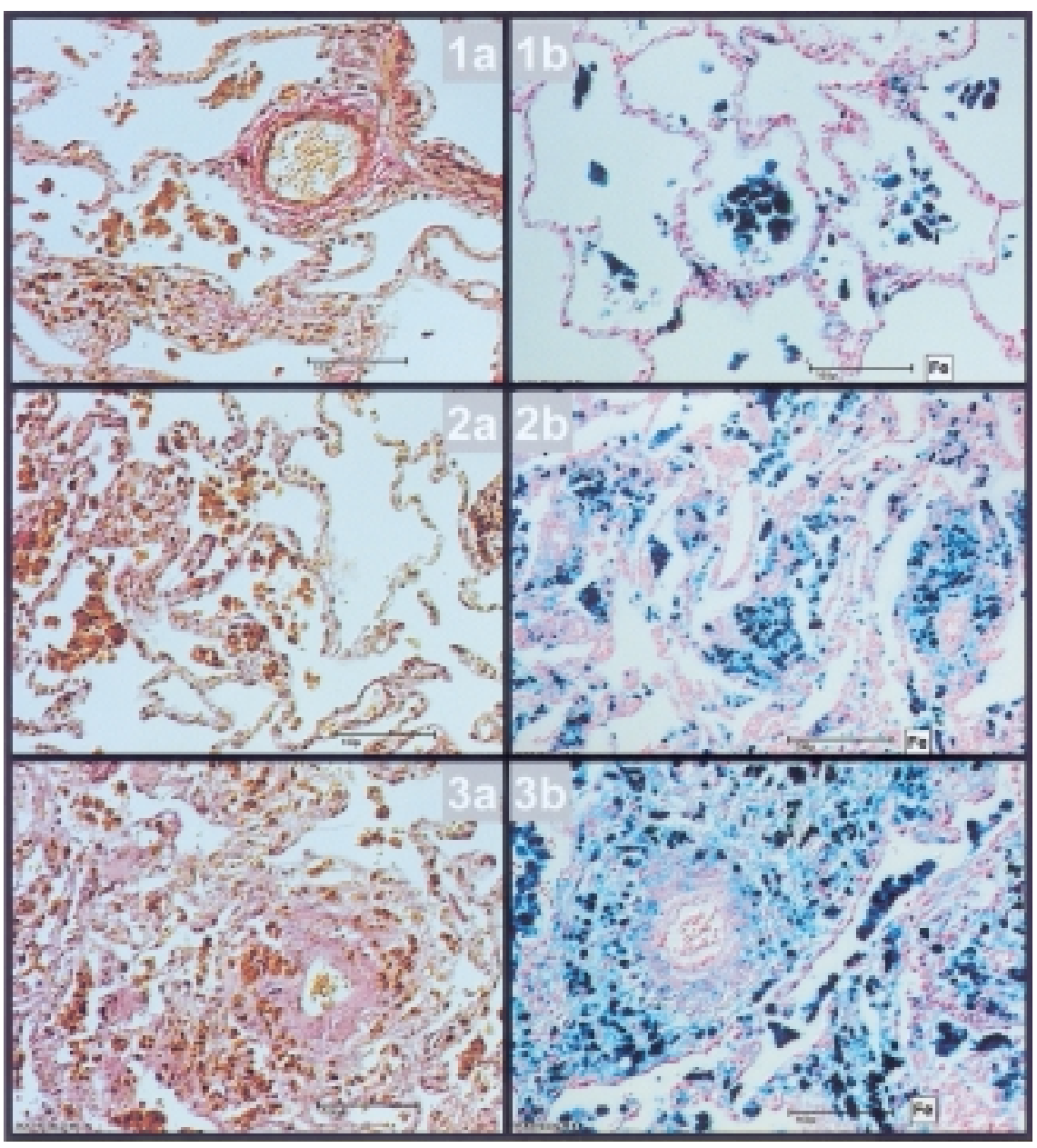

Abb. $\mathbf{1}$ a bis $\mathbf{3 b}$ Mikrofotogramme der graduell unterschiedlichen Ausprägung von Sideropneumokoniosen

1 a bis 3 a: Elastica van Gieson-Färbung $1 \mathrm{~b}$ bis $3 \mathrm{~b}$ : Berliner-Blau-Färbung

Abb. 1 a, b Grad I der Schweißerlunge. Schweißarbeiten als Landmaschinenschlosser über 36 Jahre. Exposition gegenüber Zink, Chrom und Nickel eine Stunde pro Woche. Starker Raucher!

Abb. 2 a, b u. 3 a, b Grad II und Grad III der Schweißerlunge. 56 Jahre alter Mann. 13 Jahre Arbeiten als Schutzgasschweißer von Tankanlagen unter besonders ungünstigen Bedingungen. Schweißerlunge als BK anerkannt. 
Deutliche Zeichen einer chronisch-schwelenden, entzündlichfibrosierenden Reaktion.

Entwicklung herdförmig akzentuierter, den Fremdstoffdepots topographisch zugeordneter Lungenfibrosen (Abb. 3a,b).

Unter Berücksichtigung dieser morphologischen Kriterien für die Graduierung erfolgt die Zuteilung, wobei zusammenfassend alle in dem jeweiligen Präparat vorhandenen Veränderungen Berücksichtigung finden. In dem ausgewerteten Untersuchungsgut konnten bei 7 Patienten der Grad I, bei 21 Patienten der Grad II und bei 10 Patienten der Grad III zusammenfassend festgelegt werden (Abb.4).

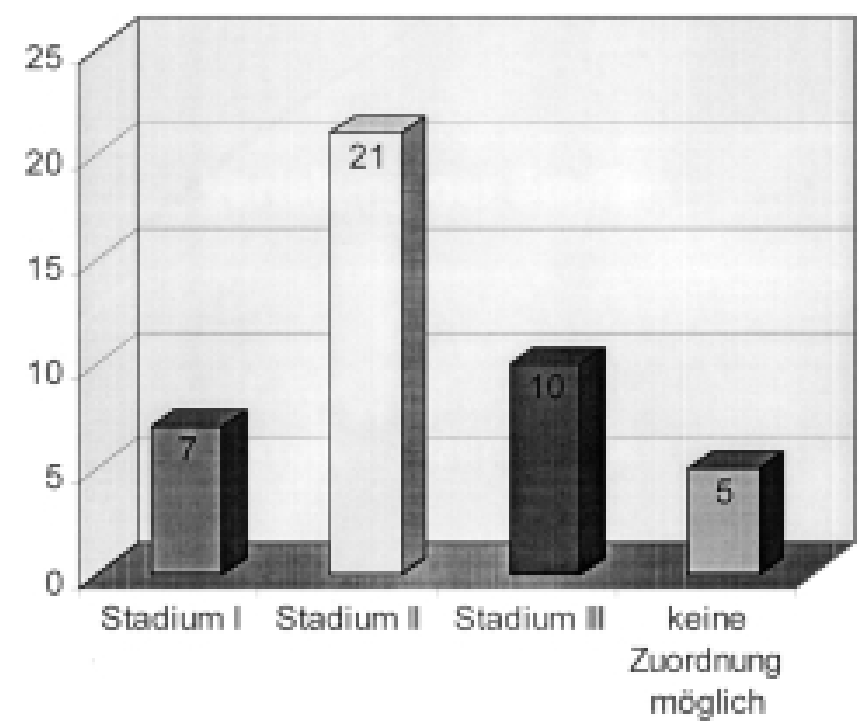

Abb. 4 Zuordnung der Befunde von 43 Exponierten nach morphologisch fassbaren Schweregraden von Sideropneumokoniosen. Keine nennenswerten Befunde in fünf Gewebsproben.

Bei 5 Patienten sind trotz der Anamnese einer beruflichen Schweißrauchexposition wesentliche Befunde im Sinne einer Sideropneumokoniose nicht zu belegen. Dabei ist allerdings zu berücksichtigen, dass in diesen Fällen nur relativ kleine transbronchial gewonnene Präparate von 1 bis $2 \mathrm{~mm}$ Durchmesser zur Verfügung standen.

\section{Diskussion}

Die Untersuchungen von Gewebsproben von 43 Patienten unter Berücksichtigung der Angaben unterschiedlicher beruflicher Expositionen gegenüber Schweißrauchen haben ergeben, dass durchaus histomorphologisch und histochemisch unterschiedliche Schweregrade der unter dem Oberbegriff der Sideropneumokoniosen zusammengefassten Befunde $\mathrm{zu}$ dokumentieren sind. Bei der Zuordnung der Befunde müssen allerdings auch Reaktionsmuster auf weitere chronische inhalative Noxen wie Rauchgewohnheiten Berücksichtigung finden. Die Abgrenzung der zellulären und fibrosierenden Reaktionen als Folge der Schweißrauchinhalation zu Reaktionen auf chronisches Inhalationsrauchen im Sinne der Kondensatpneumopathie kann im Einzelfall schwierig sein.

Die Untersuchungsergebnisse zeigen aber, dass bei entsprechenden Anamnesen eine meist beruflich bedingte nennens- werte Schweißrauchexposition zu pathologisch-anatomisch fassbaren Lungenveränderungen führen kann, die durchaus zu pathologischen Befunden mit Krankheitswert im Sinne einer Sideropneumokoniose führen können. Allein die histopathologischen Befunde erlauben aber keinen definitiven Rückschluss über die im Einzelfall zu folgernden objektivierbaren funktionellen Rückwirkungen auf Atmung und Lungenkreislauf. Besonders zu beachten ist, dass bei der Untersuchung verschiedener Präparate desselben Patienten und sogar innerhalb eines Präparates unterschiedliche Schweregrade der Reaktionsmuster vorliegen können. Diese Befunde bei Auswertung umfangreicheren Untersuchungsgutes bedingen eine kritische Bewertung der durchaus eindrucksvollen morphologischen Befunde besonders dann, wenn nur vergleichsweise kleine, z.B. transbronchial gewonnene Biopsiepräparate zur Verfügung stehen. Die Einteilung verschiedener Schweregrade einer sogenannten Schweißerlunge kann also nur bei Kombination der histologischen Befunde mit den klinisch, radiologisch und funktionsanalytisch fassbaren Befunden sowie den arbeitsanamnestischen Angaben erfolgen. Allein basierend auf der histomorphologischen Graduierung der Stadien I bis III einer Schweißerlunge sind konkrete Rückschlüsse auf Art und Schweregrad der Einschränkung der Lungenfunktion besonders auch unter versicherungsmedizinischen Aspekten nicht definitiv möglich.

\section{Literatur}

${ }^{1}$ Barth J, Höltmann B, Müller KM. Alveolar-septale Fibrose und systemische Eisenbelastung bei einem Schweißer. Atemw.-Lungenkrkh 1986; 7: 290-293

2 Morgenroth K, Verhagen-Schröter G. Licht- und elektronenmikroskopische Untersuchungen und energiedispersive Röntgenmikroanalyse an Biopsiematerial zur Pathogenese der Schweißerlunge. Atemw.-Lungenkrkh 1984; 10: 451 - 456

${ }^{3}$ Rösler JA, Morgenroth K, Müller KM, Woitwitz HJ. Lungenfibrosen nach langjähriger Einwirkung von Schweißrauchen. Verh Dtsch Ges Arbeitsmed 1995; 35: 285-288

${ }^{4}$ Buckup H. Die Schweißerlunge - differente oder indifferente Lungenveränderungen. Zentralbl Arbeitsmed 1973; 23: 286293

${ }^{5}$ Radenbach D, Nowak D, Jörres R, Magnussen H. Schweißen und Atmungsorgane. Atemw.-Lungenkrkh 1995; 2: 105-111

${ }^{6}$ Rösler JA, Woitowitz HJ. Spiroergometrie bei einem Schweißer mit Hypertonus, chronischer obstruktiver Atemwegserkrankung und Lungenfibrose. Atemw.-Lungenkrkh 1999; 4: 208-214

${ }^{7}$ Verhoff MA, Müller KM. Sideroelastose der Pulmonalgefäße nach Schweißrauchexposition. Pathologe 2000; 21: 229-233

${ }^{8}$ Müller KM, Grewe P. Pathologie der Pneumokoniosen. Atemw.Lungenkrkh 1992; 10: $428-436$

${ }^{9}$ Zschische W. Schweißerlunge - ein einheitliches Krankheitsbild?. In: Konietzko N, Costabel U, Bauer PC (Hrsg.). Lunge und Arbeitswelt. Berlin: Springer Verlag, 1990: 123-147

\section{Prof. Dr. med. K. M. Müller}

Institut für Pathologie der Bergbau-Berufsgenossenschaft an den Berufsgenossenschaftlichen Kliniken Bergmannsheil Universitätsklinik Ruhr-Universität Bochum Bürkle-de-la-Camp-Platz 1 44789 Bochum 